\title{
Tradurre Céline, a cura di Gianfranco Rubino. Atti del Convegno dell'Università di Cassino
}

\section{Emanuele Kanceff}

\section{(2) OpenEdition}

1 Journals

\section{Edizione digitale}

URL: https://journals.openedition.org/studifrancesi/46252

DOI: 10.4000/studifrancesi.46252

ISSN: 2421-5856

\section{Editore}

Rosenberg \& Sellier

\section{Edizione cartacea}

Data di pubblicazione: 1 octobre 2007

Paginazione: 474-475

ISSN: 0039-2944

\section{Notizia bibliografica digitale}

Emanuele Kanceff, «Tradurre Céline, a cura di Gianfranco Rubino. Atti del Convegno dell'Università di Cassino», Studi Francesi [Online], 152 (LI | II) | 2007, online dal 30 novembre 2015, consultato il 24 novembre 2021. URL: http://journals.openedition.org/studifrancesi/46252 ; DOI: https://doi.org/ 10.4000/studifrancesi.46252

Questo documento è stato generato automaticamente il 24 novembre 2021.

\section{(c) (†) $\odot$}

Studi Francesi è distribuita con Licenza Creative Commons Attribuzione - Non commerciale - Non opere derivate 4.0 Internazionale. 


\title{
Tradurre Céline, a cura di Gianfranco Rubino. Atti del Convegno dell'Università di Cassino
}

\author{
Emanuele Kanceff
}

\section{NOTIZIA}

AA. vV., Tradurre Céline, a cura di Gianfranco RUBINo. Atti del Convegno dell'Università di Cassino, Cassino, Università, Dipartimento di Linguistica e Letterature comparate, 2001 (Collana "dimore", 1), pp. 176.

1 Questo confronto su Céline, apparentemente di ordine linguistico, ma in realtà fortemente orientato da opzioni comparatistiche, si rivela di grande interesse a una attenta lettura. Denso di significati, nonostante la relativamente piccola mole del libro, sarebbe difficilmente sintetizzabile in poche frasi e nel breve spazio che ci è concesso. Non rinunciamo, tuttavia, a dettagliarne i contenuti.

Gianfranco RUBINo, nella sua Premessa, ricorda la giusta prevenzione di Céline per le traduzioni e si chiede quale livello, quale immagine percepisca dalle traduzioni il lettore non francofono, quanto possa recepire del funambolismo verbale che caratterizza l'opera celiniana, rilevando come quello delle traduzioni di Céline sia un terreno estremo di sperimentazione. Henri GODARD (Qu'est-ce que le style de Céline?) parte dalla constatazione della varietà infinita degli effetti di stile e dei mezzi che li producono in Céline e ne dimostra la diversità e la paradossale unità attraverso un passo di Féerie pour une autre fois. Studiando le discontinuità e le apparenti incoerenze del brano, Godard ne mette in rilievo i principi sotterranei che ne fanno uno stile d'autore fra i più nuovi e più potenti del suo secolo. Jacqueline RISSET, D'un chateau de mots l'autre, rileva gli effetti straordinari che l'incontro di delirio e lucidità produsse nella sua scrittura. La parabola della scrittura celiniana e la conseguente contraddittoria e imbarazzata parabola della critica le sono di campo d'indagine per 
concludere sul vero significato della trasposizione e dello spostamento, che non sono geografici. Gli chateaux celiniani sono castelli di parole, «edificati pe catturare il vuoto, per spiare il passaggio dal cosmo al caos». Elio NASUELLI, L'involontario: poetica e stile nelle corrispondenze celiniane degli anni d'esilio, parte dalla constatazione che, con l'edizione Pléiade-Einaudi, l'insieme dei romanzi è praticamente disponibile, mentre rimane scoperto l'insieme delle corrispondenze, materiale immenso in attesa di sistemazione. Traduzione a parte, «si tratta di ritrovare nei testi cosiddetti occasionali o minori le costanti di una scrittura, gli stilemi che si presentano anche al grado di elaborazione meno ordito possibile: il livello spontaneo di uno stile». Lavorando su questa ipotesi e mostrando il grado di disarticolazione sintattica dei suoi scritti, l'A. definisce la "frase ritmica", le unità enunciative isolate che divengono frasi grazie al ritmo. La conclusione è che il progetto di Céline è stato quello di inventare una lingua espressiva oltre i codici e le convenzioni grammaticali, un "attentato letterario alle norme del letterario» che ha reso altamente ardua la sua ricezione tra gli scrittori del Novecento. Jacques CELLARD, Le problème de l'argot: quelques considérations, si chiede in primis che cosa sia l'argot per poi ragionare su quello di Céline e sul come tradurlo, esemplificando sul suo uso e notando come esso diminuisca progressivamente nei testi dello scrittore francese a profitto di una pura e semplice invenzione verbale. Valerio MAGRELLI, Il tema della traduzione nella metafora dello "style-métro», si riferisce ai nuovi studi sul tema della ferrovia nelle letterature europee, precisando che il confronto avviene tra una realtà sociale e un codice linguistico, non tra un'innovazione tecnologica e un'esperienza concreta. Queste premesse gli permettono di entrare nel vivo dei testi celiniani sullo stesso tema, con una necessaria riflessione delle affinità fra treno e métro e sulle ragioni di scelta che hanno orientato lo scrittore. «Come versare un'opera in una nuova lingua, si chiede Magrelli, senza modificare la curva ferroviaria del racconto, la parabola della sua corsa, lo scarto del treno narrativo?», concludendo sulla sfida impossibile della traduzione che il testo non pare replicabile. Franco BIJFFoNI, Céline e le nuove frontiere della traduttologia, considera la posizione celiniana dell'unicità e irriproducibilità dell'opera come espressione di un idealismo oggi inattuale, affermando che la moderna traduttologia propone di considerare il testo letterario «non come un rigido scoglio immobile nel mare, bensì come una piattaforma galleggiante, dove chi traduce opera sul corpo vivo dell'opera, ma l'opera stessa è in costante movimento». Il testo si muove verso il futuro all'interno delle incrostazioni della lingua, ma anche verso il passato, se si tiene conto degli avantesti. Lino GABELLONE, Inventare la lingua. Su Céline, tra lettura e traduzione, considera l'invenzione della lingua come esigenza poetica prima, «insieme defondatrice e fondatrice, rottura e superamento», soprattutto nei momenti cruciali dell'evoluzione formale. Il tentativo celiniano di intensificazione espressiva, tentativo di far vivere una lingua morta almeno per un po', avviene «attraverso una ricomposizione della lingua in una stratificazione complessa che non può essere ridotta al solo "parlato basso" ma che accoglie e riesce ad amalgamare registri molto diversi». In questo processo il lettore perde il senso puntuale del linguaggio per sostituirlo con un ritmo e una mobilità necessari. Il traduttore, anche lui lettore, si muove «nella scomoda posizione di dover trovare non solo un equivalente della lingua di Céline... ma di dover inventare un tono lavorando insieme sulla prossimità e sulla distanza, sulla familiarità e lo straniamento. Céline impone al traduttore anche una anamnesi della propria lingua parlata e il recupero di un orecchio insieme linguistico e musicale». Quale che sia il risultato, l'esperienza di scoprire Céline traducendolo ha il potere di far discendere nelle zone germinative di una lingua che si sta inventando. 
3 Anna JERONIMIDIS, L'argot e i suoi traduttori, dopo aver ritracciato in breve la storia delle traduzioni italiane di Céline, di cui dà in appendice una folta bibliografia, riflette sull'idea di argot in Céline.

Ernesto FERRERO, Tradurre la «petite musique», analizza vari esempi di traduzioni per giungere alla conclusione che il lavoro del traduttore è ontologicamente esposto alla frustrazione e allo scacco di fronte alla musica intraducibile del testo originale.

5 Annie OLIVER, Les étapes de "Voyage". Traduire et retraduire, esamina le traduzioni italiane del "Voyage", i problemi che ne derivano e le polemiche relative.

6 Marie HÉDIARD, Les niveaux de la langue à l'épreuve de la traduction: le cas du "que" célinien dans "Voyage au bout de la nuit", si occupa, con consumata esperienza teorica, di un problema del tutto particolare, il valore e il funzionamento del que in francese.

7 Paolo CARILE, Amarcord, ci offre, attraverso ricordi personali, uno spaccato della difficile e controversa ricezione dello scrittore in Italia.

8 Pier Carlo BONTEMPELL, Osservazioni sulle traduzioni e sulla recente ricezione di Céline in Germania, si avventura su un terreno ancora più difficile e pericoloso, quello della ricezione in Germania. Infine, Filippo LA PORTA, Viaggi organizzati al termine della notte. Céline e la nuova narrativa italiana, lancia uno sguardo sui territori del presente per scoprire che la nostra narrativa migliore è consapevole, come lo era Céline, della doppia natura di menzogna e sortilegio propria del linguaggio, ma si ostina ad abbellire il vuoto: come se dal viaggio fino al termine della notte abbia trovato non il buio ma uno schermo sempre acceso, senza trovare il linguaggio idoneo ad esprimere questa esperienza. 